\title{
Vasos Subclavios: ¿Qué tan cerca se Encuentran de la Clavícula?
}

\section{Subclavian Vessels: How Close are They to the Clavicle?}

\author{
Daniel P. Manosalvas ${ }^{10}$ Claudio A. Calvo ${ }^{2}$ \\ Anselmo I. Alegria ${ }^{4}$ Rodrigo J. Liendo ${ }^{2}$ \\ ${ }^{1}$ Residente del Programa de Formación de Especialista de Ortopedia y \\ Traumatología de la Universidad Católica de Chile, Santiago, Chile \\ 2 Médico Traumatólogo, Equipo de Hombro, Departamento de \\ Ortopedia y Traumatología, Universidad Católica de Chile, Santiago, \\ Chile \\ ${ }^{3}$ Médico Traumatólogo, Departamento de Ortopedia y \\ Traumatología, Universidad de Chile, Santiago, Chile \\ ${ }^{4}$ Interno de Medicina, Escuela de Medicina, Universidad Católica de \\ Chile, Santiago, Chile
}

Rev Chil Ortop Traumatol 2019;60:39-46.

\author{
Francisco J. Soza ${ }^{2}$ Camila P. Azócar ${ }^{3(0)}$
}

\begin{abstract}
Address for correspondence Camila Azócar Sanhueza, MD, Camino el Parque 100, dept. 2803, edificio Alerce, Vitacura, Santiago, Chile (e-mail: camiazocars@gmail.com).
\end{abstract}

\section{Resumen}

\section{Palabras claves}

- vasos subclavios

- arteria subclavia

- lesión vascular

- cirugía de hombro

- fractura de clavícula
Introducción la lesión de los vasos subclavios durante la cirugía de clavícula es una situación rara, de suceder podría resultar incluso mortal; conocer su ubicación es indispensable para minimizar ese riesgo.

Objetivo determinar la ubicación y la distancia de la AS y VS respecto a la clavícula. Secundariamente, identificar las características particulares que influencien la ubicación y la distancia.

Materiales y Método estudio retrospectivo, AngioTAC de tórax y cuello entre 2012 y 2017; se midió la longitud de la clavícula, distancia y dirección de los vasos subclavios en cada tercio de la clavícula, como también la angulación entre una horizontal y el centro de los vasos subclavios.

Resultados 39 AngioTC, 78 hombros. Distancia AS/clavícula tercio proximal, medio y distal $32,8 \mathrm{~mm} \quad(20,3-46,3), \quad 15,4 \mathrm{~mm} \quad(6,8-28,0)$ y $62,7 \mathrm{~mm} \quad(37,0-115,4)$ respectivamente. La distancia VS/clavícula tercio proximal, medio y distal fue: $7,4 \mathrm{~mm}(1,0-19,2), 16,2 \mathrm{~mm}(6,7-34,7)$ y $67,1 \mathrm{~mm}(29,7-117,0)$ respectivamente. La ubicación de AS y VS con respecto a la clavícula es posterosuperior en el tercio proximal, posteroinferior en el tercio medio e inferior en el tercio distal.

Conclusión En el tercio proximal la vena puede estar solo a $1 \mathrm{~mm}$ de la clavícula y la arteria a $6 \mathrm{~mm}$ en dirección antero-posterior, resultando esa zona la más peligrosa. En el tercio medio la distancia es mayor, pudiendo estar arteria y vena a solo $6 \mathrm{~mm}$, la dirección de brocado más peligrosa es antero-inferior con una inclinación promedio de $45^{\circ}$ caudal. El tercio distal es el más seguro, los vasos están al menos a $30 \mathrm{~mm}$ de distancia hacia caudal.

Nivel de evidencia III. received

January 3, 2019

accepted

July 24, 2019
DOI https://doi.org/

10.1055/s-0039-1696710. ISSN 0716-4548.
Copyright $\odot 2019$ by Thieme Revinter

Publicações Ltda, Rio de Janeiro, Brazil
License terms

(ㄷ) (i) $\ominus$ (5) 


\begin{abstract}
Background injury to the subclavian vessels during clavicle surgery is a rare situation, if it happens it could even be fatal; knowing their location is essential to minimize that risk.

Objective determine location and distance of the AS and VS with respect to the clavicle. Secondarily identify particular characteristics that influence location and distance.

Materials and Methods retrospective study, AngioTAC of thorax and neck between 2012 and 2017; it was measured the length of the clavicle, distance and direction of the subclavian vessels in each third of the clavicle and angulation between a horizontal and the center of the subclavian vessels were measured.

Results 39 AngioTC, 78 shoulders. AS / clavicle third proximal, middle and distal distance $32.8 \mathrm{~mm}$ (20.3-46.3), $15.4 \mathrm{~mm} \quad(6.8-28.0)$ and $62.7 \mathrm{~mm} \quad(37.0-115.4)$ respectively. Distance VS / clavicle third proximal, middle and distal was: $7.4 \mathrm{~mm}$ (1.0-19.2), $16.2 \mathrm{~mm}(6.7-34.7)$ and $67.1 \mathrm{~mm}(29.7-117.0)$ respectively. The location of AS and VS with respect to the clavicle is posterosuperior in the proximal third, posteroinferior in the middle third and inferior in the distal third.

Keywords

- subclavian vessels

- subclavian artery

- vascular injury

- shoulder surgery

- clavicle fracture

Conclusion In the proximal third the vein can be only $1 \mathrm{~mm}$ from the clavicle and the artery to $6 \mathrm{~mm}$ in the anterior-posterior direction, this zone is the most dangerous. In the middle third the distance is greater, artery and vein can be only to $6 \mathrm{~mm}$, the most dangerous drilling direction is antero-inferior with an average inclination of $45^{\circ}$ caudal. The distal third is the safest, the vessels are at least $30 \mathrm{~mm}$ away from the vessels.

Level of evidence III.
\end{abstract}

\section{Introducción}

La fractura de clavícula es una patología de alta frecuencia, y representa entre el $2 \%-5 \%{ }^{1-3}$ de todas las fracturas y entre el $35 \%-44 \%$ de las fracturas de la extremidad superior en los adultos. $^{2}$

En la actualidad, el manejo de la fractura de clavícula desplazada y conminuta es habitualmente quirúrgico con el fin de disminuir el riesgo de no unión y mal unión sintomática, ${ }^{4-6}$ donde aparece la reducción abierta y osteosíntesis con placa como la técnica de elección para ese tipo de fracturas. ${ }^{7}$

Ese procedimiento tiene los riesgos propios de cualquier cirugía $^{8,9}$ y se suman las complicaciones específicas de la técnica quirúrgica dentro de las cuales se encuentra la lesión de estructuras vasculares, que en ese caso, corresponde a la lesión de la Arteria Subclavia (AS) y de la Vena Subclavia (VS). ${ }^{10}$

Las lesiones vasculares se pueden producir en dos momentos distintos. Lesiones inmediatas: aquellas que se producen durante la cirugía, la VS es la que acumula más lesiones de ese tipo; pueden ser al momento de instalar el implante, del brocado y la manipulación de la fractura, su probabilidad aumenta en la medida que aumenta el tiempo de evolución, se debe prestar especial atención a la manipulación del callo óseo. ${ }^{8}$ Lesiones a largo plazo: la AS resulta lesionada como resultado de tornillos prominentes que contactan el vaso generando pseudoaneurismas, los que pueden presentarse años después de la cirugía y generalmente se manifiestan como isquemia crítica de la extremidad. ${ }^{11-13}$

Si bien las lesiones de esas estructuras son poco frecuentes, ${ }^{8}$ corresponden a complicaciones graves que pueden incluso comprometer la vida del paciente. ${ }^{14-16}$ Por esa razón es importante durante la cirugía y también después, reducir al mínimo el riesgo de daño de esas estructuras; y la mejor forma de lograrlo es conocer la ubicación exacta de los vasos subclavios y así tomar las precauciones del caso durante el procedimiento quirúrgico. $^{17,18}$

El objetivo de este trabajo, es describir la ubicación de los vasos subclavios con respecto a la clavícula. Como objetivos secundarios, se busca describir las diferencias según lateralidad y sexo, e identificar diferencias propias de muestra de población.

\section{Materiales y Método}

Estudio retrospectivo, revisión de fichas clínicas y registro imagenológico, previa aprobación del comité de ética de nuestra institución. Se realizaron mediciones de Angiotomografía Computada (AngioTAC) de tórax y de extremidades superiores con los hombros en rotación neutra y los brazos a los lados del tórax, estudios realizados entre enero del 2012 y diciembre del 2017 de nuestra institución, que además tuvieran imágenes en formato DICOM, de la historia clínica se registró edad, sexo y antecedentes patológicos, el examen se realizó por sospecha de patología vascular de cuello o extremidad superior, se incluyeron solo aquellos en los que se descartó patología.

Se excluyeron pacientes con antecedente de fracturas alrededor del hombro, trauma torácico, patología tumoral, trombosis, presencia de aneurisma, catéter, marcapasos, diagnóstico confirmado de síndrome de opérculo torácico 
o exámenes solicitados por enfermedad vascular de la extremidad superior. Se excluyeron también pacientes con esqueleto inmaduro.

Usando reconstrucciones sagitales perpendiculares al eje de la clavícula, se midieron de forma bilateral, las distancias y la dirección entre la clavícula y la arteria subclavia y vena subclavia. La longitud de la clavícula se midió en relación con su eje mayor; se dividió en 3 partes iguales: tercio proximal, medio y distal. En cada tercio se marcó un punto central: puntos: A, B y C (-Fig. 1).

La distancia entre clavícula y vasos subclavios se midió en un corte sagital perpendicular al eje de la clavícula en relación con los puntos A, B y C; en dicho corte se trazó una línea desde la cortical de la clavícula que se encontraba más próxima al vaso a ser medido hasta la pared de la vena o arteria respectivamente (ej. en el punto A desde la cortical posterior de la clavícula hasta la pared anterior de la AS) (-Fig. 2).

Para determinar la dirección, se midió la angulación entre una horizontal que pasa por el punto central de cada segmento (A, B y C) y el centro del vaso evaluado usando el mismo corte sagital utilizado para medir distancia (-Fig. 3). Los valores negativos se consideraron como dirección cefálica del vaso con respecto a la clavícula y los valores positivos como dirección caudal.

Para el análisis estadístico, se utilizó el programa STATA (StataCorp 2011; Stata Statistical Software: release 14, StataCorp, College Station, TX, USA). Para el análisis de variables no paramétricas, se utilizaron medianas y rangos con test Mann-Whitney. Para las medias se utilizó el test de Wilcoxon para datos pareados. Las variables categóricas se describen como frecuencias relativas.

\section{Resultados}

Se evaluaron 86 AngioTAC, 39 exámenes cumplieron con los criterios de inclusión y exclusión; 7 hombres y 32 mujeres, se midieron un total de 78 hombros.
La posición tanto de la vena como de la arteria subclavia con respecto a la clavícula, presentó un cambio progresivo desde proximal a distal desde una posición posterosuperior en el punto A, luego posteroinferior en el punto $\mathrm{B}$ y finalmente inferior en el punto $C$.

La mediana de distancia desde la clavícula hasta la arteria en el punto A fue de $32,8 \mathrm{~mm}$ (rango 20,3 a 46,3); en el punto B fue de 15,4mm (rango 6,8 a 28,0); y en el punto $C$ fue de $62,7 \mathrm{~mm}$ (rango 37,0 a 115,4 ). No se encontraron diferencias significativas al analizar por sexo ni lateralidad (-Fig. 4.).

La posición de AS con respecto a la clavícula en el tercio proximal (punto A) tuvo una mediana de $17,7^{\circ}$ en dirección cefálica con un rango desde - $63,7^{\circ}$ cefálico a $8,4^{\circ}$ caudal, posición predominantemente posterosuperior (-Fig. 5a.). En el tercio medio (punto B) fue de $46,8^{\circ}$ caudal (rango $25,8^{\circ}$ a $74,8^{\circ}$ caudal) (-Fig 6a.) y en el tercio distal (punto C) fue de $86,3^{\circ}$ caudal (rango $73,2^{\circ}$ a $99,7^{\circ}$ caudal) (-Fig 7a.).

La mediana de la distancia desde la clavícula hasta la vena en el punto A fue de 7,4mm (rango 1,0 a 19,2); en el punto B fue de $16,2 \mathrm{~mm}$ (rango 6,7 a 34,7); y en el punto $C$ fue de $67,1 \mathrm{~mm}$ (rango 29,7 a 117,0). La distancia de la VS a nivel del tercio proximal, fue significativamente más cercana para mujeres $(p=0,04)$ independiente de su lateralidad ( - Fig 8.).

La posición de la vena con respecto a la clavícula en el tercio proximal (punto A) fue de $-6,1^{\circ}$ en dirección cefálica con un rango desde $-38,1^{\circ}$ cefálico a $33,3^{\circ}$ caudal ( - Fig 5b.). En el tercio medio (punto B) fue de $69,3^{\circ}$ caudal $\left(9,9^{\circ}\right.$ a $88,9^{\circ}$ caudal) ( - Fig 6b.) y en el tercio distal (punto $\mathrm{C}$ ) fue de $84,2^{\circ}$ caudal $\left(71,1^{\circ}\right.$ a $99,5^{\circ}$ caudal) (-Fig 7b.). Sin diferencias por sexo ni lateralidad.

En la figura 4 se muestran los gráficos de resumen de la distribución de las mediciones de distancia y angulación de los vasos subclavios con respecto a la clavícula.

La mediana de largo de la clavícula fue de 145,5mm (rango 124,3 a 164,3). En hombres fue más larga que en mujeres, sin encontrarse diferencia estadística, con una mediana de $154,7 \mathrm{~mm}$ (rango 143,0 a 164,3 ) y $141,9 \mathrm{~mm}$ (rango 124,3 a $158,0)$ respectivamente.

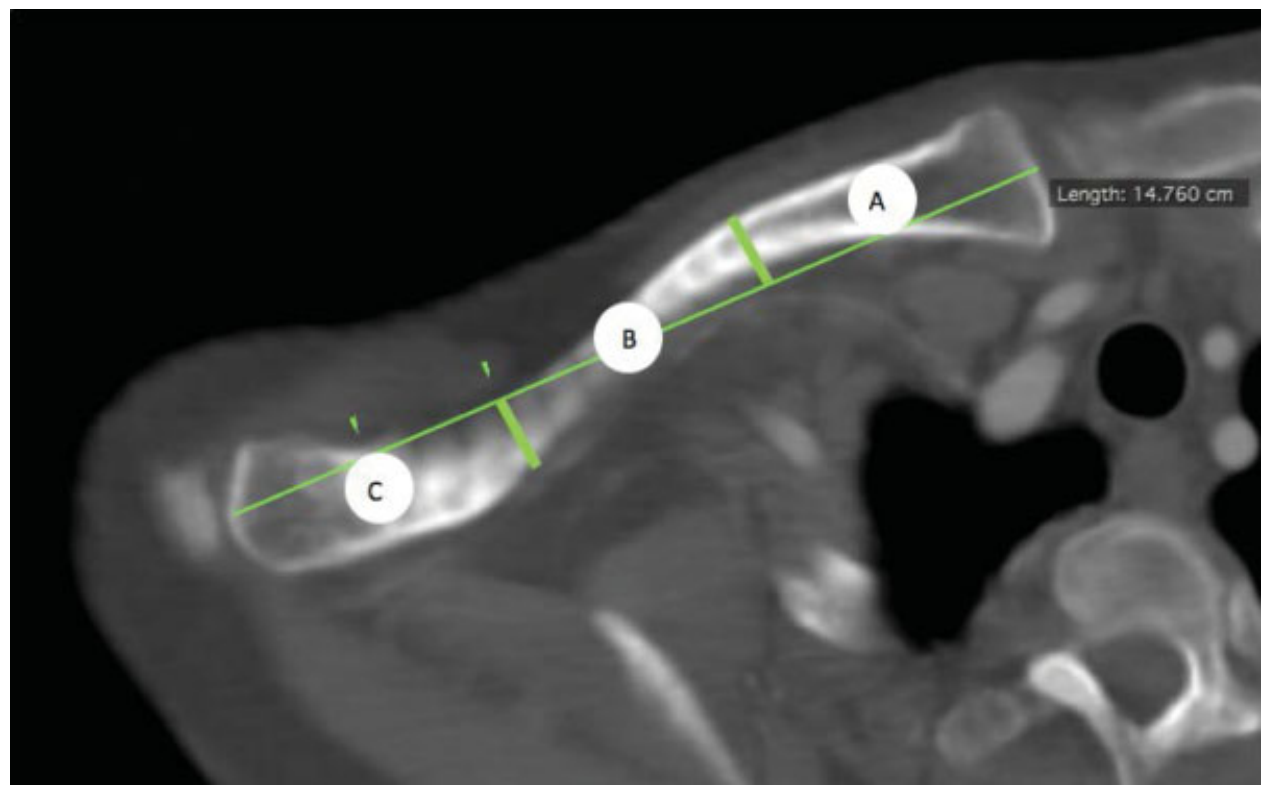

Fig. 1 AngioTAC de hombro, corte axial de clavícula. Medición de largo de la clavícula y determinación de puntos centrales A, B y C. 


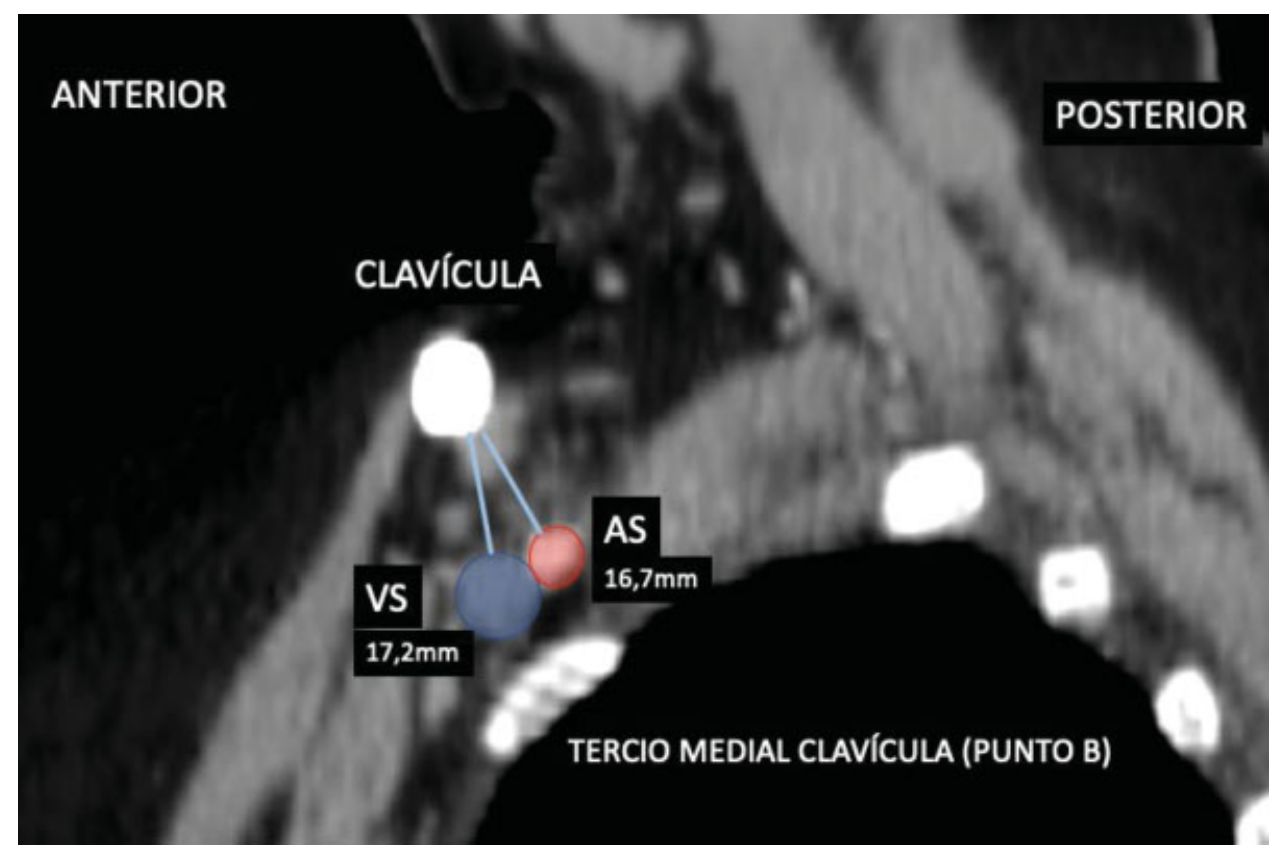

Fig. 2 AngioTAC de hombro, corte sagital perpendicular al eje clavícula en el punto B. Medición de la distancia entre la clavícula y arteria subclavia (rojo) y vena subclavia (azul)

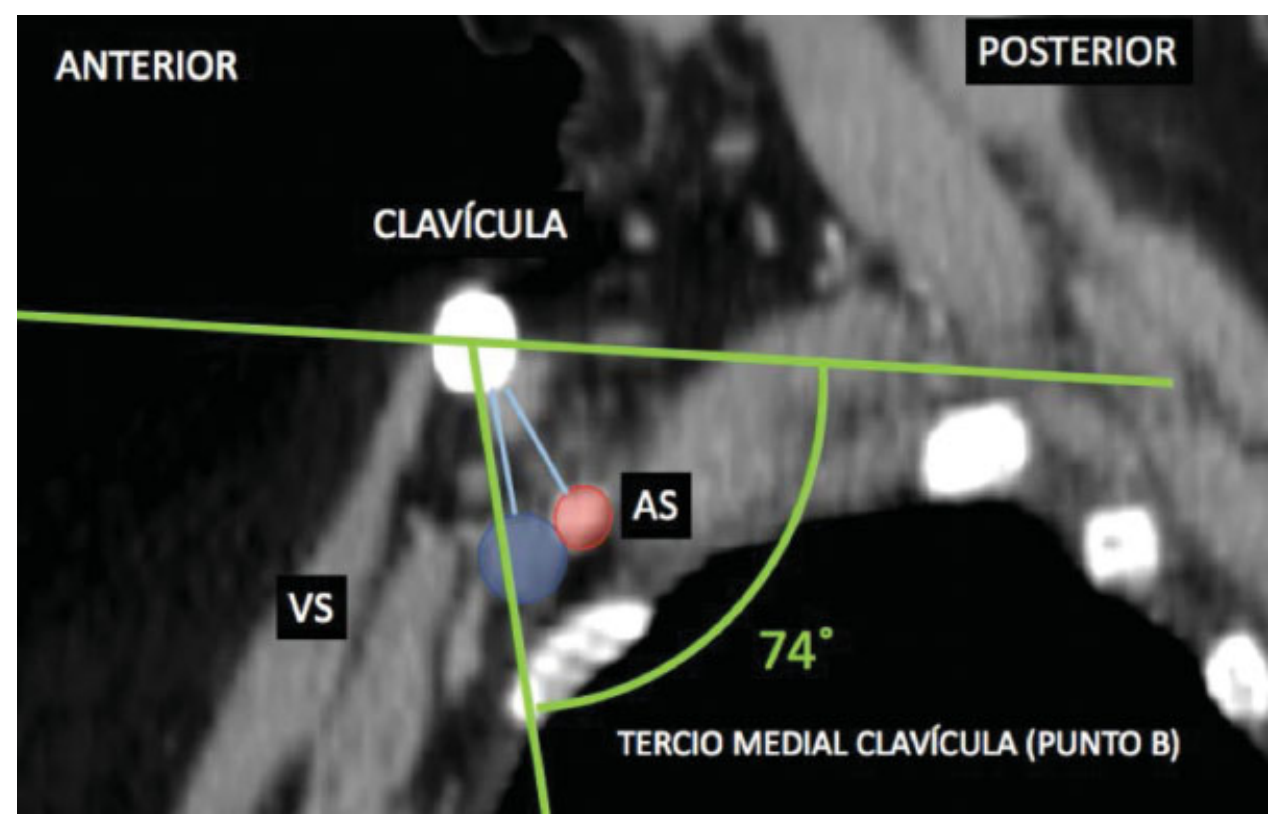

Fig. 3 AngioTAC de hombro, corte sagital perpendicular al eje clavícula en el punto B. Medición de ángulo entre clavícula y arteria subclavia (rojo) y vena subclavia (azul).

\section{Discusión}

El tratamiento quirúrgico de la fractura de clavícula utilizando osteosíntesis con placa y tornillos es común, considerado técnicamente "sencillo", sin embargo, no exento de complicaciones debido a la cercanía de estructuras vitales, y de no ser ejecutada adecuadamente puede resultar en lesiones que supongan la perdida de la funcionalidad de la extremidad superior e incluso la vida el paciente. ${ }^{9}$ Algunas complicaciones pueden presentarse años después de la cirugía inicial.
Al momento de tratar quirúrgicamente una fractura de clavícula, los vasos subclavios están en constante riesgo de lesión debido a su cercanía con la clavícula, ellos se pueden lesionar por manipulación de los fragmentos óseos, brocado para la fijación con material de osteosíntesis o tornillos prominentes. Por esas razones, se hace imperativo conocer la dirección y distancia de los vasos subclavios y las zonas seguras para disminuir al mínimo el riesgo de daño al brocar, controlando la dirección y la profundidad, además de no dejar tornillos prominentes. 


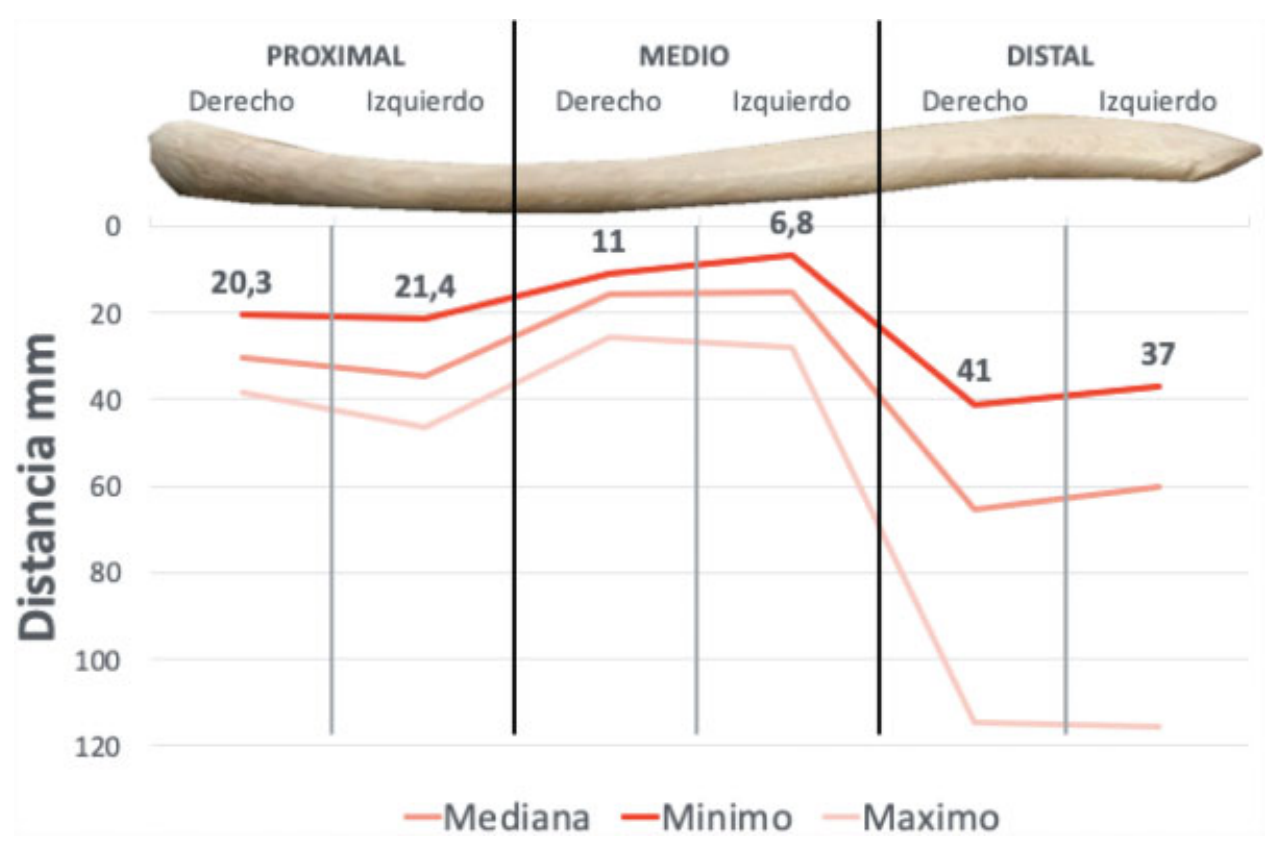

Fig. 4 Distancia entre clavícula y arteria subclavia según tercio. Mínima, mediana y máximo.

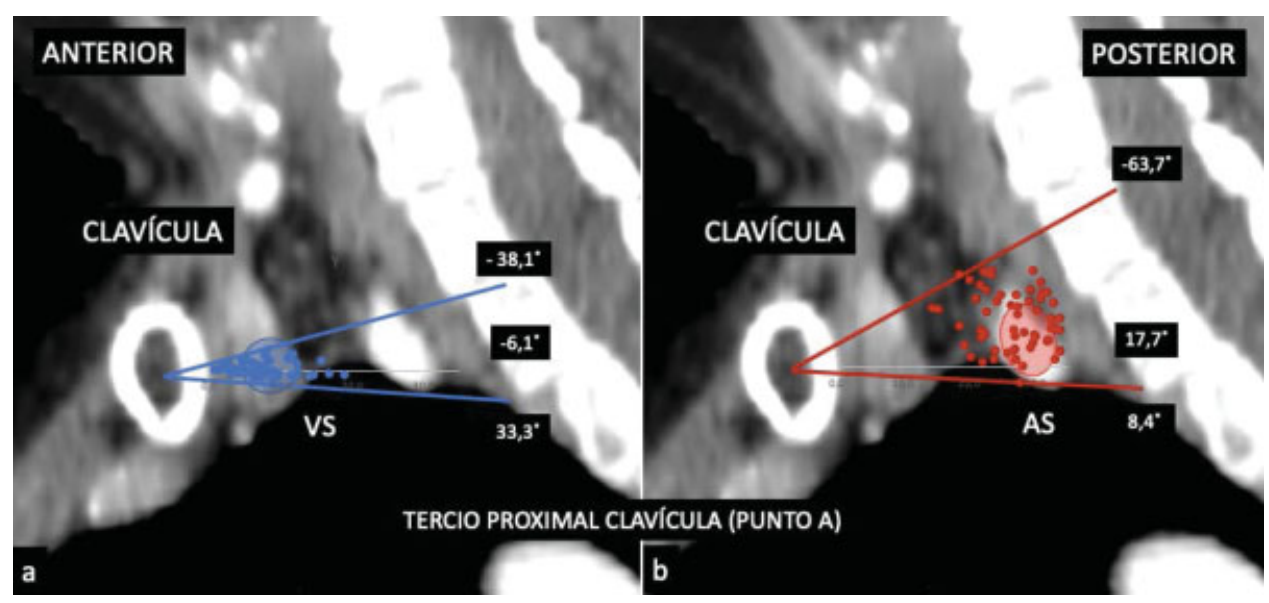

Fig. 5 Angulación entre clavícula de arteria (a) y vena subclavia (b) en relación con la horizontal en el punto A.

Basándonos en nuestros resultados y los de la literatura respecto a ese tema, podemos concluir que el tercio proximal es el sitio de mayor riesgo de lesión de estructuras vasculares dado que ambos vasos subclavios se encuentran en extrema cercanía a la cortical de la clavícula, incluso encontrándose en algunos casos a $1 \mathrm{~mm}$ de distancia en el caso de la VS, en cuanto a la dirección de brocado resulta más peligroso hacerlo de anterior a posterior dada la posición posterosuperior de los vasos. Considerando la dirección y la distancia de los vasos, surgen dos recomendaciones, primero, evitar brocar de anterior a posterior, preferir hacerlo de arriba hacia abajo y segundo, de tener que hacerlo, recomendamos brocar para colocar tornillos unicorticales.

El tercio medio de la clavícula, según nuestros resultados, es un sitio de riesgo intermedio, la dirección de mayor peligro es la posteroinferior, con inclinación promedio de 45 grados caudal, si bien la distancia promedio es de 15 milímetros puede estar a tan solo 6 milímetros, el uso de un protector (ej. separador Hohmann), es una medida a tomar en cuenta. Otra consideración técnica, podría ser el uso de placas anteriores que es mucho más segura que una placa superior, con eso, la dirección del brocado se haría hacia una zona segura, nuevamente la prominencia de los tornillos debe ser lo menor posible, los vasos subclavios se acercan aún más durante los movimientos del hombro especialmente sobre la cabeza, pudiendo llevar a daño a largo plazo. En ese segmento, recomendamos usar placas anteriores que usan brocado de anterior a posterior; colocar un separador ya sea por detrás o por debajo de la clavícula; finalmente, y para controlar mejor la profundidad del brocado, usar brocas de buena calidad, una broca en malas condiciones puede duplicar la distancia. ${ }^{19}$

El tercio distal de la clavícula se muestra como el sitio más seguro con respecto a los vasos subclavios que se encuentran alejados al menos 30 milímetros y en posición inferior, de hecho, es más probable lesionar el paquete neurovascular 


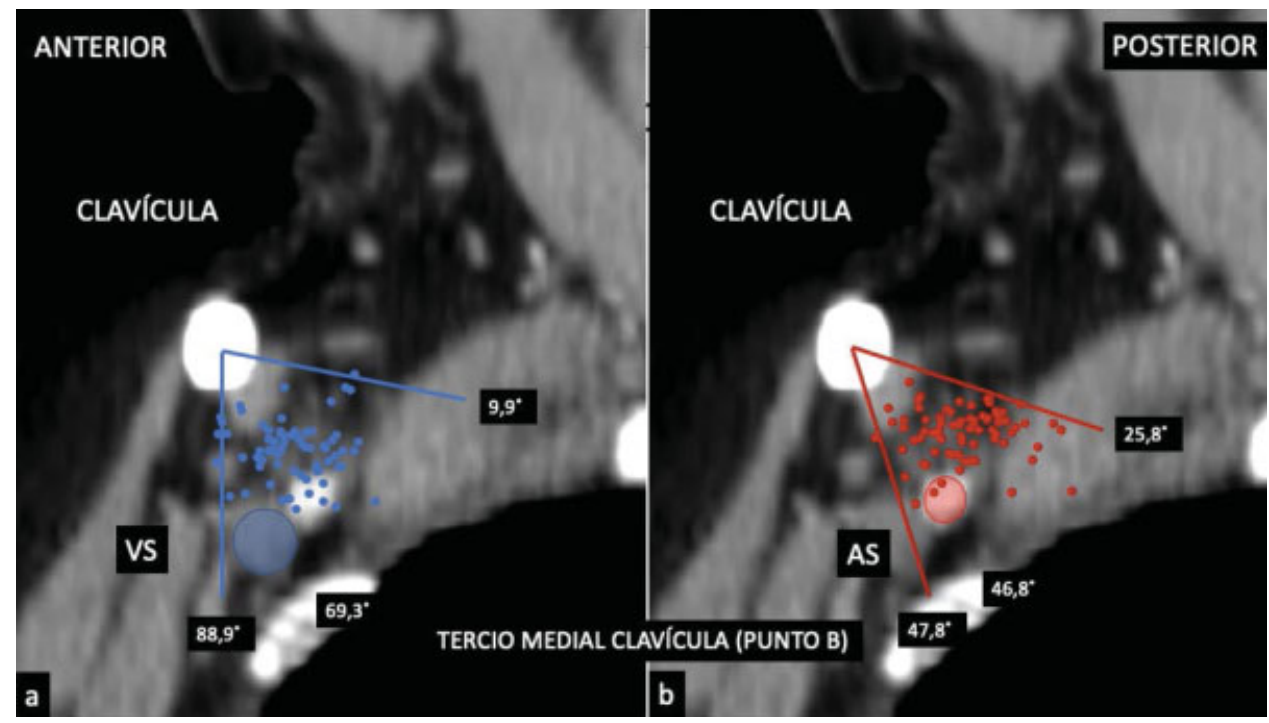

Fig. 6 Angulación entre clavícula de arteria (a) y vena subclavia (b) en relación con la horizontal en el punto B.

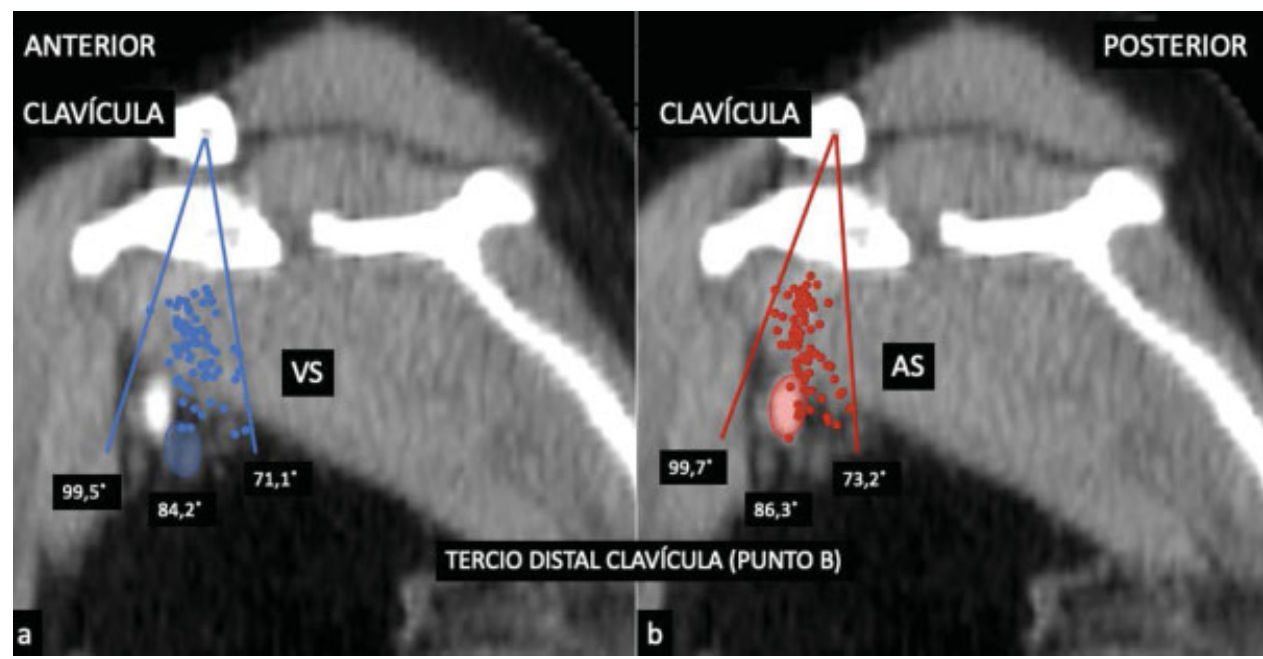

Fig. 7 Angulación entre clavícula de arteria (a) y vena subclavia (b) en relación con la horizontal en el punto C.

supraescapular, por eso cuidar la profundidad de brocado, incluso en esa zona, no es algo para descuidar.

Nuestros resultados son comparables con los encontrados por Sinha y col. ${ }^{20}$ en su trabajo encontró que los vasos subclavios se encuentran más cerca a la clavícula en su tercio proximal siendo la VS la estructura más próxima a la cortical clavicular en ese segmento. Una debilidad del trabajo de Sinha es la selección de pacientes, pues incluyeron en las mediciones pacientes con patología vascular activa al momento de la medición; en nuestro caso, esos pacientes fueron excluidos. Una diferencia destacable en nuestro estudio fue una menor distancia $(2 \mathrm{~mm})$ significativa de los vasos subclavios con respecto a la clavícula en pacientes mujeres por lo que es importante tener especial cuidado al intervenir mujeres, Esa diferencia podría ser el resultado de una mayor cantidad de mujeres en nuestra serie.

El presente estudio es el primero en describir la posición de los vasos subclavios con respecto a la clavícula en población chilena y sudamericana; además pudimos aumentar el número de pacientes evaluados en comparación a los estudios internacionales. Creemos también que, al haber usado una muestra con criterios de inclusión y exclusión más estrictos, los resultados son más representativos. Finalmente, al no encontrar diferencias importantes con los resultados obtenidos por trabajos en poblaciones norteamericanas y europeas, la interpretación futura de trabajos realizados en esas poblaciones, es más extrapolable a nuestros pacientes, Desde luego, hacen falta más estudios para afirmar eso con soporte estadístico.

\section{Conclusión}

La dirección y distancia entre clavícula y vasos subclavios es constante entre hombres y mujeres, sin diferencia significativa entre el lado derecho e izquierdo, y no se ve influenciada por el tamaño del paciente. En orden de riesgo de mayor a menor está el tercio proximal, medio y distal. Los vasos describen una curva detrás de la clavícula desde proximal que se sitúan posterior y algo cefálico hasta situarse completamente caudal en el tercio distal. 


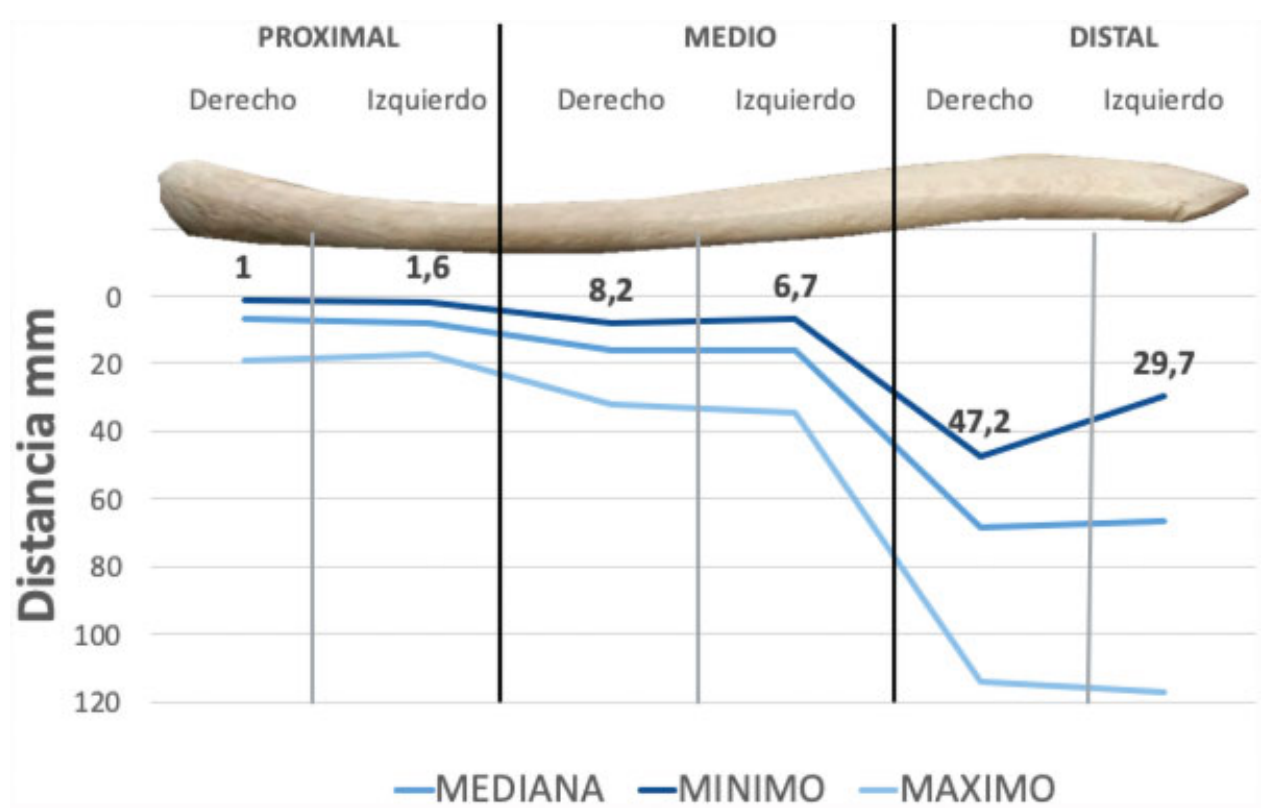

Fig. 8 Distancia entre clavícula y vena subclavia según tercio. Mínimo, mediana y máximo.

En el tercio proximal, la vena se encuentra a tan solo un milímetro de la cortical posterior y la arteria a 6 milímetros considerando una dirección de brocado de anterior a posterior, resultando esa zona la más peligrosa. En el tercio medio, la distancia es algo mayor tanto la arteria como la vena al menos a 6 milímetros. Para ese segmento, la dirección de brocado más peligrosa es anterorinferior con una inclinación promedio de 45 grados caudal. En el tercio distal es seguro brocar en cualquier dirección, los vasos están al menos a 30 milímetros de distancia en dirección caudal.

No olvidar que en la práctica clínica, los pacientes excluidos de este trabajo potencialmente serán nuestros pacientes. Planificar adecuadamente, considerando situaciones que puedan poner aún más cerca los vasos, evitará que se produzcan lesiones.

\section{Conflictos de Interés}

Los autores declaran no tener conflicto de interés en el presente trabajo.

\section{Bibliografía}

1 Oh JH, Kim SH, Lee JH, Shin SH, Gong HS. Treatment of distal clavicle fracture: a systematic review of treatment modalities in 425 fractures. Arch Orthop Trauma Surg 2011;131(04):525-533

2 Singh R, Rambani R, Kanakaris N, Giannoudis PV. A 2-year experience, management and outcome of 200 clavicle fractures. Injury 2012;43(02):159-163

3 Postacchini F, Gumina S, De Santis P, Albo F. Epidemiology of clavicle fractures. J Shoulder Elbow Surg 2002;11(05):452-456

4 Uzer G, Yildiz F, Batar S, Bozdag E, Kuduz H, Bilsel K. Biomechanical comparison of three different plate configurations for comminuted clavicle midshaft fracture fixation. JShoulder Elbow Surg 2017;26(12):2200-2205

5 van der Meijden OA, Gaskill TR, Millett PJ. Treatment of clavicle fractures: current concepts review. J Shoulder Elbow Surg 2012; 21(03):423-429
6 Burnham JM, Kim DC, Kamineni S. Midshaft Clavicle Fractures: A Critical Review. Orthopedics 2016;39(05):e814-e821

7 Nourian A, Dhaliwal S, Vangala S, Vezeridis PS. Midshaft Fractures of the Clavicle: A Meta-analysis Comparing Surgical Fixation Using Anteroinferior Plating Versus Superior Plating. JOrthop Trauma 2017;31(09):461-467

8 Wijdicks FJG, Van der Meijden OA, Millett PJ, Verleisdonk EJ, Houwert RM. Systematic review of the complications of plate fixation of clavicle fractures. Arch Orthop Trauma Surg 2012;132 (05):617-625

9 Clitherow HD, Bain GI. Major neurovascular complications of clavicle fracture surgery. Shoulder Elbow 2015;7(01):3-12

10 Bain GI, Galley IJ, Keogh AR, Durrant AW. Axillary artery pseudoaneurysm after plate osteosynthesis for a clavicle nonunion: A case report and literature review. Int J Shoulder Surg 2010;4(03):79-82

11 Clitherow HDS, Bain GI. Association between screw prominence and vascular complications after clavicle fixation. Int J Shoulder Surg 2014;8(04):122-126

12 Gullo J, Singletary EM, Larese S. Emergency bedside sonographic diagnosis of subclavian artery pseudoaneurysm with brachial plexopathy after clavicle fracture. Ann Emerg Med 2013;61(02): 204-206

13 van Schaik PM, de Borst GJ, Moll FL, Toorop RJ. Late onset acute occlusion of the subclavian artery after clavicle fracture. Vascular 2015;23(06):661-662

14 Bain G, Eng K, Zumstein M. Fatal Air Embolus During Internal Fixation of the Clavicle: A Case Report. Vol. 3, JBJS Case Connector. 2014:24-24

15 Shackford SR, Connolly JF. Taming of the screw: a case report and literature review of limb-threatening complications after plate osteosynthesis of a clavicular nonunion. JTrauma 2003;55(05): 840-843, discussion 843

16 Bain GI, Eng K, Zumstein MA. Fatal Air Embolus During Internal Fixation of the Clavicle: A Case Report. JBJS Case Connect 2013;3 (01):e24

17 Hematti H, Mehran RJ. Anatomy of the thoracic duct. Thorac Surg Clin 2011;21(02):229-238, ix

18 Urschel HC Jr. Anatomy of the thoracic outlet. Thorac Surg Clin 2007;17(04):511-520 
19 Stillwell A, Ioannou C, Daniele L, Tan SLE. Osteosynthesis for clavicle fractures: How close are we to penetration of neurovascular structures? Injury 2017;48(02): 460-463
20 Sinha A, Edwin J, Sreeharsha B, Bhalaik V, Brownson P. A radiological study to define safe zones for drilling during plating of clavicle fractures. JBone Joint Surg Br 2011;93(09): $1247-1252$ 